\title{
Controller Design for the Rotational Dynamics of a Quadcopter
}

\author{
RABIA RASHDI* ZEESHAN ALI**, JAVED RAHMAN LARIK***, LIAQUAT ALI JAMRO****, AND \\ UROOJ BAIG*
}

\section{RECEIVED ON 29.05.2018 ACCEPTED ON 30.10.2018}

\begin{abstract}
Researchers have shown their interests in establishing miniature flying robots to be utilized for, both, commercial and research applications. This is due to that fact that there appears to be a huge advancement in miniature actuators and sensors which depend on the MEMS (Micro Electro-Mechanical Systems) NEMS (Nano-Electro Mechanical Systems). This research underlines a detailed mathematical model and controller design for a quadcopter. The nonlinear dynamic model of the quadcopter is derived from the Newton-Euler method and Euler Lagrange method. The motion of a quadcopter can be classified into two subsystems: a rotational subsystem (attitude and heading) and translational subsystem (altitude and $x$ and $y$ motion). The rotational system is fully actuated whereas translational subsystem is under actuated. However, a quadcopter is 6 DOF (Degrees of Freedom) under actuated system. The controller design of a quadcopter is difficult due to its complex and highly nonlinear mathematical model where the state variables are strongly coupled and contain under actuated property. Nonlinear controller such as SMC (Sliding Mode Controller) is used to control altitude, yaw, pitch, and roll angles.Simulation results show that the robustness of the SMC design gives a better way to design a controller with autonomous stability flight with good tracking performance and improved accuracy without any chattering effect. The system states are following the desired trajectory as expected.
\end{abstract}

Key Words: Proportional-Integral-Derivative, Linear Quadric Regulator, Sliding Mode Controller, Degrees of Freedom, Vertical Take-Off and Landing.

\section{INTRODUCTION}

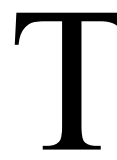

he UAVs (Unmanned Aerial Vehicles) were initially introduced during the World War-I. The advancement of UAVs has produced awesome enthusiasm for the programmed control region in these most recent couple of decades. Recently, Quadcopters have attracted many researchers because it can replace humans in accomplishing dangerous tasks.
Various linear and nonlinear control techniques such as fuzzy logic, LQR (Linear Quadric Regulator), PID (ProportionalIntegral-Derivative) and feedback linearization have been used to control the quadcopter. Santos et. al. [1] worked on a smart fuzzy controller for a quadcopter. A fuzzy logic control system was designed and applied for controlling the dynamic model of a quadcopter. Simulation results show that the

Authors E-Mail: rabbiarashdi@yahoo.com, zeeshan.ali@faculty.muet.edu.pk, javedlarik@gmail.com, engr_laj@yahoo.com, uroojbaig1992@gmail.com)

* Department of Mechatronics Engineering, Mehran University of Engineering \& Technology, Jamshoro, Pakistan.

** Department of Industrial Engineering \& Management, Dawood University of Engineering \& Technology, Karachi, Pakistan.

*** Department of Mechanical Engineering, Mehran University of Engineering \& Technology, Jamshoro, Pakistan.

**** Academics \& Training, Sindh Technical Education and Vocational Training Authority, Tano Jam, Pakistan.

This is an open access article published by Mehran University Research Journal of Engineering and Technology, Jamshoro under the CC by 4.0 International License. 
efficiency of this smart controller is satisfactory. However, the main drawback of this research was that its controller design was too complex. $\mathrm{Li}$ and $\mathrm{Li}$ [2] presented his model to examine the dynamic response and PID control algorithm for a quadcopter. This study elucidates the construction and dynamic model of a quadcopter. Apart from this, the study also proposed a controller which aims to control the orientation and position of the 6DOF model. DOF can be defined as the maximum number of independent variables, the independent motion states in this case, which a quadcopter model can execute. Their model shows instability if the values of PID controller gains such as proportional gain $(\mathrm{Kp})$, integral gain $(\mathrm{Ki})$ and derivative gain $(\mathrm{Kd})$ are not compatible with each other. These gains can be tuned to acquire the desired response of the system. Salih et.al. controlled the quadcopter system using PID controller technique. In this paper, a state-space model was illustrated and for the ease of simulation the nonlinear mathematical models were altered to the Simulink based models [3]. Dief and Yoshida [4] solved asymmetry problem that is usually results from the motor. They also compared the responses of the quadcopter between classical controller such as PID and adaptive control. Peddle et. al. [5] proposed a model which controls the altitude, velocity, and position of a ductfan for VTOL using PI controller. In this paper, a large scale four-rotors machine was designed and instead of using electric motor, they used 04 gasoline engines to generate thrust force. This nature of engine speed variation resulted in an unstable system due to the gasoline engines. Inaccuracy, uncertainty and nonlinearity of the system modeling are additionally the foremost problems. Hence, a flexible and a robust control design is needed. For nonlinear systems, sliding mode control is designed. Seshagiri and Promtun [6] computed the angle of attack command using sliding mode controller based on model linearization. Two types of sliding surfaces were compared; the $1^{\text {st }}$ order and the $2^{\text {nd }}$ order and their experimental results proved that the performance of the $2^{\text {nd }}$ order SMC was better. Koshkouei et. al. [10] presented dynamic and higher order sliding mode control technique. Conditions for reaching to appropriate sliding surface were proposed in this paper. Herrera et. al. [7] described the application and control design of the quadcopter using SMC from a PD (Proportional-Derivative) sliding surface. However, some disturbances are included in this paper. Heba presented the detailed mathematical model of a quadcopter and different control approaches were used: PID, SMC, Back-stepping controller and Gain scheduling based PID controller [8]. The simulation showed huge degradation when the system was exposed to wind. In this study, overshoot and chattering effect occurred during translation and rotational maneuvering. Wang et. al. [11] proposed a cascade PID feedback control algorithm to stabilize the attitude of a quadcopter. Doukhi et. al. [12] designed a robust Self-Tuning PID controller based on fuzzy logic to adjust the external disturbances produced by the changes in the payload weight during the flight period.

The objective of this paper is to design an SMC based controller to control the rotational dynamics of a quadcopter. The dynamics and kinematics of a quadcopter are developed using the Newton-Euler formalism and Euler-Langrange equations which incorporates the suitable criteria. The ultimate goal is to reduce the response time and to reduce the overshoot in the previously presented literature. Reducing the chattering effect in the SMC is another factor which is covered in the controller design.

\section{QUADCOPTER MATHEMATICAL MODEL}

The dynamics of the quadcopter are nonlinear and has 6DOF (03 rotational and 03 translational) and 04 inputs which makes the system under-actuated. Under-actuated systems are those which have less control inputs than system's DOF. A quadcopter has simple dynamic features; it has four rotors which are connected in one-end cross frame. Every rotor has a propeller mounted to an individually operated BLDC motor. Propeller 1 and 3 spin in clockwise direction and propeller 2 and 4 spin in anticlockwise direction to balance the moments and canceling the gyroscopic as well as aerodynamics torques during the steady flight.

Mehran University Research Journal of Engineering \& Technology, Volume 38, No. 2, April, 2019 [p-ISSN: 0254-7821, e-ISSN: 2413-7219] 
The dynamics and kinematics of the quadcopter are derived from the research work presented in [8-9]. In the overall mathematical modeling, the following assumptions are made to preserve the simplicity of the system.

(1) The design frame is symmetrical and rigid.

(2) The CG of the quadcopter overlaps with the origin of body-fixed frame.

(3) Rigid propellers.

(4) Drag force and thrust force are function of the square of the speed of the propellers.

There are two reference frames acting on a quadcopter as shown in Fig. 1. The Earth-frame having N (North), E (East) and D (downward) axis and the body-frame with $\mathrm{x}$, $\mathrm{y}$, and $\mathrm{z}$ axis.

The body and inertial frame can be related by rotation matrix as:

$R=\left[\begin{array}{ccc}c \theta c \psi & s \phi s \theta s \psi & c \phi s \theta c \psi+s \phi s \psi \\ c \theta s \psi & s \phi s \theta s \psi+c \theta c \psi & c \phi s \theta s \psi-s \theta c \psi \\ -s \theta & s \phi c \theta & c \phi c \theta\end{array}\right]$

The rotation matrix is used to define the dynamics of quadcopter and represents the movement of the quadrotor model from the earth to the space.

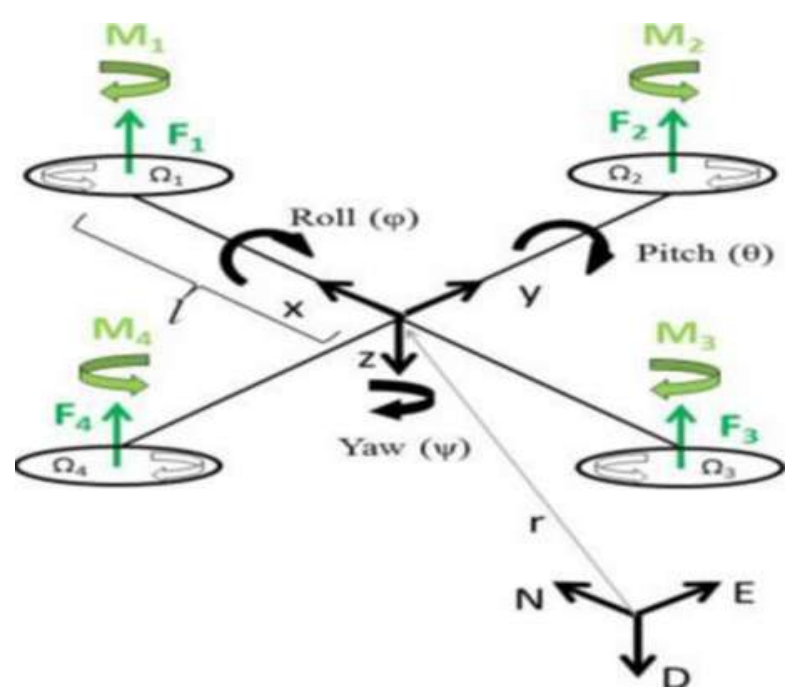

FIG. 1. SCHEMATIC DIAGRAM OF A QUADCOPTER
The quadcopter has twelve states defined as:

$$
X=[\phi \dot{\phi} \theta \dot{\theta} \psi \dot{\psi} z \dot{z} x \dot{x} y \dot{y}]
$$

The state-vector describes the position of the quadcopter in space and it's linear as well as angular velocities.

The equations of motions are obtained using force and moment balance:

$$
\begin{aligned}
& \ddot{\phi}=\frac{l}{I_{x x}} U_{2}-\frac{J_{r}}{I_{x x}} \dot{\theta} \Omega_{r}+\frac{I_{y y}}{I_{x x}} \dot{\psi} \dot{\theta}-\frac{I_{z z}}{I_{x x}} \dot{\theta} \dot{\psi} \\
& \ddot{\theta}=\frac{l}{I_{y y}} U_{3}-\frac{J_{r}}{I_{y y}} \dot{\phi} \Omega_{r}+\frac{I_{z z}}{I_{y y}} \dot{\psi} \dot{\theta}-\frac{I_{x x}}{I_{y y}} \dot{\psi} \dot{\phi} \\
& \ddot{\psi}=\frac{l}{I_{z z}} U_{4}+\frac{I_{z z}}{I_{z z}} \dot{\theta} \dot{\phi}-\frac{I_{y y}}{I_{z z}} \dot{\phi} \dot{\psi} \\
& \ddot{x}=\frac{-U_{1}}{m}(\sin \phi \sin \psi+\cos \phi \cos \psi \sin \theta) \\
& \ddot{y}=\frac{-U_{1}}{m}(\cos \phi \sin \psi \sin \phi-\cos \psi \sin \phi) \\
& \ddot{z}=g-\frac{U_{1}}{m}(\cos \phi \cos \theta)
\end{aligned}
$$

Where the input signal $U_{1}$ represents total thrust, $U_{2}, U_{3}$ and $\mathrm{U}_{4}$ represent roll, pitch and yaw moments, $\mathrm{m}$ is the quadcopters's mass, $\mathrm{J}_{\mathrm{r}}$ defines the inertia of each rotor and, $I_{x x}, I_{y y}$ and $I_{z z}$ are quadcopter's inertia in $x, y$, and $z$ longitudinal positions respectively.

The required input signals from Equations (3-8) are defined as:

$$
\begin{aligned}
& U_{1}=K_{f}\left(\Omega_{1}^{2}+\Omega_{2}^{2}+\Omega_{3}^{2}+\Omega_{4}^{2}\right) \\
& U_{2}=K_{f}\left(-\Omega_{2}^{2}+\Omega_{4}^{2}\right) \\
& U_{3}=K_{f}\left(\Omega_{1}^{2}-\Omega_{3}^{2}\right) \\
& U_{4}=K_{M}\left(\Omega_{1}^{2}-\Omega_{2}^{2}+\Omega_{3}^{2}-\Omega_{4}^{2}\right)
\end{aligned}
$$




\section{CONTROLLER DESIGN}

SMC is a nonlinear control technique and it has remarkable properties due to its robustness, accuracy and implementation. SMC is type of VSC (Variable-Structure Controller) which uses a fast changing control law to drive the state trajectories to take after specified, userdefined surface in the state space and maintains the statetrajectories on this state. SMC objective consists of two parts as per Equation (13) the first one is corrective control part which adjust changes of the state trajectories from sliding surface it and the second part is equivalent control where time derivative of the state trajectories would remain on the sliding surface.

$\mathrm{U}_{\mathrm{t}}=\mathrm{U}_{\mathrm{c}}(\mathrm{t})+\mathrm{U}_{\text {eq }}(\mathrm{t})$

Where $\mathrm{U}_{\mathrm{t}}$ is Control law, $\mathrm{U}_{\mathrm{c}}$ is Corrective control, and $\mathrm{U}_{\mathrm{eq}}$ is Equivalent control

A block diagram of SMC is shown in Fig. 2.

In order to retain the quadcopter on the reference point despite of outer disturbances four control input equations are used. The control input $\mathrm{U}_{1}$ is used to generate altitude input whereas $\mathrm{U}_{2}, \mathrm{U}_{3}$ and $\mathrm{U}_{4}$ generates roll, pitch and yaw inputs respectively.

SMC based altitude and attitude control laws are derived as follows:

The error for the altitude is defined as:

$\mathrm{e}=\mathrm{z}-\mathrm{z}_{\mathrm{d}}$

Where $z_{d}$ is desired state and $z$ is measured state.

The sliding-surface can be expressed as:

$$
s=c_{1} e+\dot{e}
$$

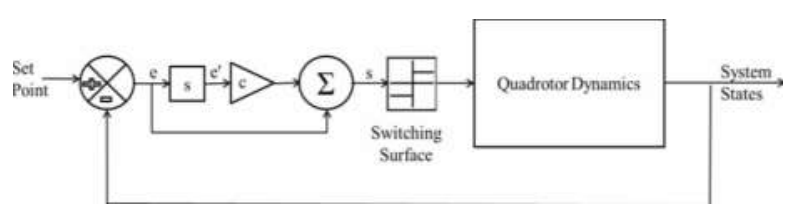

FIG. 2. BLOCK DIAGRAM OF SMC
Where $c_{1}$ is constant and is greater than zero.

By taking the derivative of the sliding surface Equation (15) with the substitution of Equation (14), the following equation is obtained:

$\dot{s}=c_{1}\left(\dot{z}-\dot{z}_{d}\right)+\ddot{z}-\ddot{z}_{d}$

The Lyapunov function is expressed as follows:

$$
V(e, s)=\frac{1}{2}\left(e^{2}+s^{2}\right)
$$

According to Lyapunov function, an exponential reaching-law has been established to follow SMC as follows:

$$
\dot{s}=k_{1} \operatorname{sgn}(s)-k_{2} s
$$

Where

$$
\operatorname{sgn}=\left\{\begin{array}{cll}
-1 & \text { if } & s<0 \\
1 & \text { if } & s>0
\end{array}\right.
$$

$\mathrm{k}_{1}$ and $\mathrm{k}_{2}$ are the design constants. Limits such as $\mathrm{k}_{1}>0$ and $\mathrm{k}_{2}<0$ have to be set on $\mathrm{k}_{1}$ and $\mathrm{k}_{2}$ to satisfy the sliding mode condition $s \dot{s}<0$. By replacing projected reachinglaw to the derivative of the sliding-surface and substituting Equation (8), the control input $U_{1}$ is obtained as Equation (18)

$U_{1}=\frac{m}{\cos \phi \cos \theta}\left[k_{1} \operatorname{sgn}(s)+k_{2} s+c_{1}\left(\dot{z}-\dot{z}_{d}\right)+g-\ddot{z}_{d}\right]$

Similarly, the same method is applied to obtain the control inputs for roll, pitch and yaw.

$$
\begin{aligned}
& U_{2}=\frac{1}{b_{1}}\left[k_{1} \operatorname{sgn}(s)+k_{2} s+c_{1}\left(\dot{\phi}_{d}-\dot{\phi}\right)+\ddot{\phi}_{d}+a_{2} \dot{\theta} \Omega_{r}-a_{1} \dot{\theta} \dot{\psi}\right] \\
& U_{3}=\frac{1}{b_{2}}\left[k_{1} \operatorname{sgn}(s)+k_{2} s+c_{1}\left(\dot{\theta}_{d}-\dot{\theta}\right)+\ddot{\theta}_{d}+a_{4} \dot{\phi} \Omega_{r}-a_{3} \dot{\phi} \dot{\psi}\right] \\
& U_{4}=\frac{1}{b_{3}}\left[k_{1} \operatorname{sgn}(s)+k_{2} s+c_{1}\left(\dot{\psi}_{d}-\dot{\psi}\right)+\ddot{\psi}_{d}-a_{5} \dot{\phi} \dot{\theta}\right]
\end{aligned}
$$

The values of $a_{1}, a_{2}, a_{3}, a_{4}, a_{5}, b_{1}, b_{2}$, and $b_{3}$ can be referred from $[8]$. 


\section{SIMULATION RESULTS AND DISCUSSION}

In this section, the response of the proposed quadcopter model with SMC based control law is simulated for various desired level of altitude (position in z-axis), desired level of roll, pitch, and yaw angles (angular position is three respective states). The physical parameters used for quadcopter are given in Table 1 [8]. The simulation is run for $2.5 \mathrm{sec}$ for each state variable which is sufficient to analyze the response of the quadcopter model under given conditions. The desired values and the respective controller gains for SMC controller are given in Table 2. These gains yield the optimum and desired response of the quadcopter as shown in Figs. 3-6. The results from previous studies show the overshoot and delayed response under the same simulation conditions [8], whereas, it can be seen that the proposed model smoothly acquires the desired altitude level (position in z-axis) as shown in Fig. 3 without any overshoot, any unnecessary delay and steady-state error. Fig. 4 depicts the desired response of the quadcopter for the given roll angle which ensures the precise tracking of the given desired signal for roll angle i.e. $5^{\circ}$. Similarly, the simulation results for pitch and yaw angles are carried out in Figs. 5-6, respectively and the proposed model executes the required tasks as expected. Moreover, the proposed model completely eliminates the chattering effect in all following simulation results. These simulation results have been validated with previous studies and are quite promising. The results have been compared with previous literatures and is observed that there is no overshoot and zero steady state error.

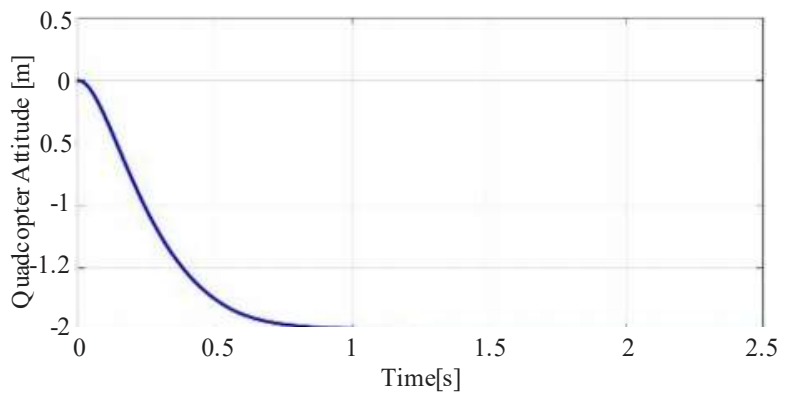

FIG. 3. ALTITUDE RESPONSE

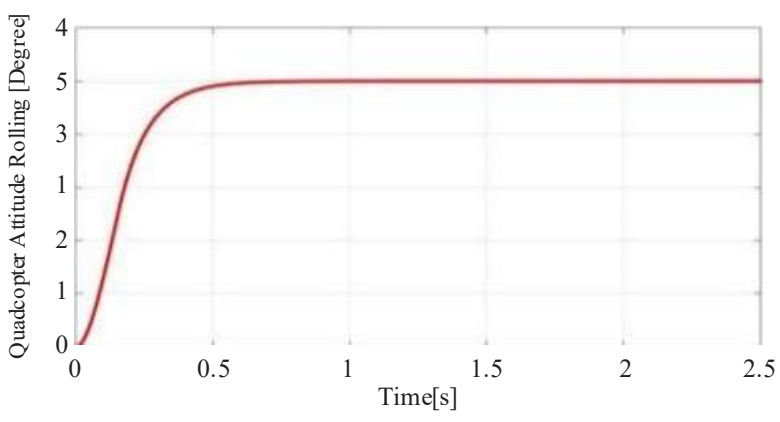

FIG. 4. ROLL RESPONSE

TABLE 1. QUADCOPTER GENERAL PARAMETERS

\begin{tabular}{|c|c|c|}
\hline Parameter & Description & Value \\
\hline $\mathrm{m}(\mathrm{kg})$ & Mass of a quadcopter & 0.65 \\
\hline $\mathrm{I}_{\mathrm{xx}}\left(\mathrm{Kg} \cdot \mathrm{m}^{2}\right)$ & Moment of inertia about body frame $\mathrm{x}$ axis & $7.5 \mathrm{e}-3$ \\
\hline $\mathrm{I}_{\mathrm{yy}}\left(\mathrm{Kg} \cdot \mathrm{m}^{2}\right)$ & Moment of inertia about body frame y axis & $1.3 \mathrm{e}-2$ \\
\hline $\mathrm{I}_{z}\left(\mathrm{Kg} \cdot \mathrm{m}^{2}\right)$ & Moment of inertia about body frame $\mathrm{z}$ axis & $6 \mathrm{e}-3$ \\
\hline $\mathrm{J}_{\mathrm{r}}\left(\mathrm{kg} \cdot \mathrm{m}^{2}\right)$ & Rotor inertia & 0.23 \\
\hline $\mathrm{l}(\mathrm{m})$ & Moment arm & 9.81 \\
\hline $\mathrm{g}\left(\mathrm{m} / \mathrm{s}^{2}\right)$ & Gravitational acceleration & $3.13 \mathrm{e}-5$ \\
\hline $\mathrm{K}_{\mathrm{f}}\left(\mathrm{N} \mathrm{s}^{2}\right)$ & Aerodynamic force constant & $7.5 \mathrm{e}-3$ \\
\hline $\mathrm{K}_{\mathrm{M}}\left(\mathrm{Nm} \mathrm{s}^{2}\right)$ & Aerodynamic moment constant & \\
\hline
\end{tabular}

TABLE 2. QUADCOPTER DESIGN PARAMATERS

\begin{tabular}{|c|c|c|c|c|}
\hline & Desired Value & $\mathrm{c}$ & $\mathrm{k}_{1}$ & $\mathrm{k}_{2}$ \\
\hline Altitude $(\mathrm{z})$ & -2 & 6.98 & 2.66 & 6.64 \\
\hline Roll and pitch $(\varnothing, \theta)$ & $5^{\circ}$ & 4.68 & 1.99 & 1.80 \\
\hline Yaw $(\psi)$ & $5^{\circ}$ & 5.24 & 1.72 & 4.33 \\
\hline
\end{tabular}

Mehran University Research Journal of Engineering \& Technology, Volume 38, No. 2, April, 2019 [p-ISSN: 0254-7821, e-ISSN: 2413-7219] 


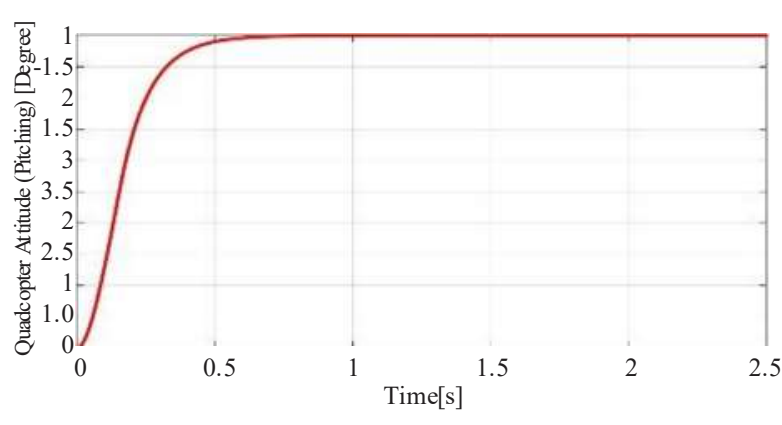

FIG. 5. PITCH RESPONSE

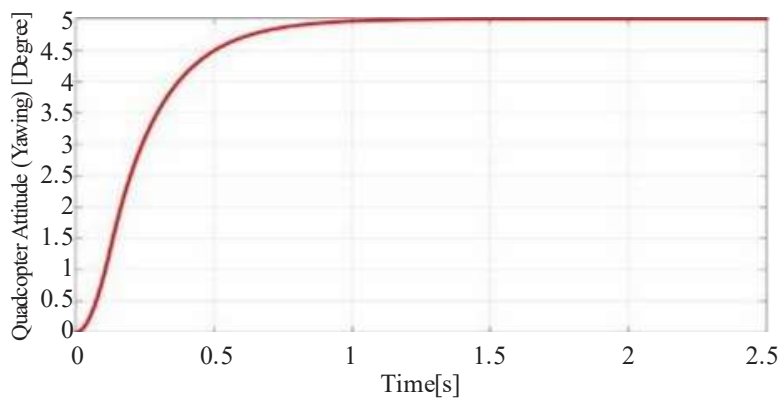

FIG. 6. YAW RESPONSE

\section{CONCLUSION}

This paper had successfully designed a controller suitable for the rotational dynamics control of a quadcopter. The mathematical model for the quadcopter was first derived using Newton-Euler formalism and Euler-Langrange equations. Sliding mode control law is used to control the altitude, roll, pitch and yaw angles of the quadcopter. The proposed mathematical model of the quadcopter is simulated for the desired state variables and the results show the improved rotational dynamic response of the system. The simulation results showed improved accuracy as compared to the previous studies without any chattering effect.

\section{ACKNOWLEDGEMENT}

The authors would like to thank the Department of Mechatronics Engineering, Institute of Information \& Communication Technologies, Mehran University of Engineering \& Technology, Jamshoro, Pakistan. Authors are also highly indebted to anonymous reviewers for their valuable comments, suggestions and diligent review.

\section{REFERENCES}

[1] Santos, M., López, V., and Morata, F., "Intelligent Fuzzy Controller of a Quadrotor", IEEE International Conference on Intelligent Systems and Knowledge Engineering, pp. 141-146, Hangzhou, 2010.

[2] Li, J., and Li, Y., "Dynamic Analysis and PID Control for a Quadrotor", IEEE International Conference on Mechatronics and Automation, pp. 573-578, Beijing, 2011.

[3] Salih, A.L., Moghavvemi, Mohamed, H.A.F., and Gaeid, K., "Flight PID Controller Design for a UAV Quadrotor", Scientific Research and Essays, Volume 5, pp. 3660-3667, December, 2010

Dief, T.N., and Yoshida, S., "Review: Modeling and Classical Controller of Quad-Rotor", International Journal of Computer Science \& Information Technology \& Security,Volume 5, No. 4, pp. 2249-555, August, 2015

Peddle, I.K., Jones, T., and Treurnicht, J., "Practical Near Hover Flight Control of a Ducted Fan (SLADe)", Control Engineering Practice, Volume 17, No. 1, pp. 48-58, January, 2009

[6] Seshagiri, S., and Promtun, E., "Sliding Mode Control of F-16 Longitudinal Dynamics", American Control Conference, pp. 1770-1775, Seattle, WA, 2008.

[7] Herrera, M., Chamorro, W., Gómez, A.P., and Camacho, O., "Sliding Mode Control: An Approach to Control a Quadrotor", Asia-Pacific Conference on Computer Aided System Engineering, pp. 314-319, Quito, 2015.

[8] Nabil, H., "Dynamic Modeling and Control of a Quadrotor Using Linear and Nonlinear Approaches", Master of Science in Robotics, Control and Smart Systems, The American University of Cairo, 2014

[9] Bouabdallah, S., "Design and Control of Quadrotors with Application to Autonomous Flying", Ph.D. Thesis, Ecole Polytechnique Feìdeìrale de Lausanne, 2007.

[10] Koshkouei, A.J., Burnham, K.J., and Zinober, A.S.I., "Dynamic Sliding Mode Control Design", IEE Proceedings of Control Theory \& Applications, Volume 152, No. 4, pp. 392-396, 8 July, 2005.

[11] Wang, P., Man, Z., Cao, Z., Zheng, J., and Zhao, Y., "Dynamics Modeling and Linear Control of Quadcopter", International Conference on Advanced Mechatronic Systems, pp. 498-503, Melbourne, VIC, 2016.

[12] Doukhi, O., Fayjie, A.R., and Lee, D.J., "Intelligent Controller Design for Quad-Rotor Stabilization in Presence of Parameter Variations", Journal of Advanced Transportation, pp. 1-10, July, 2017. 